\title{
Environmental influence on diabetes prevalence
}

Ambient air pollutants, such as fine particulate matter of $0.1-2.5 \mu \mathrm{m}$ in size $\left(\mathrm{PM}_{2.5}\right)$, may contribute to the increased prevalence of diabetes mellitus in the adult US population. " $\mathrm{PM}_{2.5}$ is known to cause morbidity and mortality through impacts on the pulmonary and systemic circulations, but the relationship with diabetes is not fully appreciated and is still under investigation," states lead author John F. Pearson, a research fellow at Harvard-MIT Health Sciences and Technology (Boston, MA, USA).

Pearson et al. examined the relationship between $\mathrm{PM}_{2.5}$ levels and the prevalence of diagnosed diabetes mellitus in the US by multivariate regression models at the county-level using data from 2004 and 2005, obtained from both the Centers for Disease Control (CDC) and the US Environmental Protection Agency (EPA).

"We added various covariates, including latitude, population density (for urbanization), exercise, obesity and others in order to rule out known diabetes risk factors in the multivariate model," explains Pearson.

Despite adjustment for over 15 covariates and various stratifications by
4 ....as $\mathrm{PM}_{2.5}$ pollution rises, so
does diabetes prevalence... 77

region and ethnicity, the investigators found a strikingly consistent and resilient association between $\mathrm{PM}_{2.5}$ and diabetes mellitus in the multiple models. The findings were further supported by the nationwide scope of the study, incorporating data from all counties in the contiguous US over 2 years.

"Although our study is ecological in nature, we clearly show that as $\mathrm{PM}_{2.5}$ pollution rises, so does diabetes prevalence," remarks Pearson. "Perhaps most troubling is that there was a $>20 \%$ difference between counties with lower levels of pollution and those with higher levels, but still below EPA limits."

The relationship between air pollutants and diabetes mellitus has been examined previously in lab experiments. Obese mice placed in heavy $\mathrm{PM}_{2.5}$ pollution (near the threshold, but below EPA limits) demonstrate an increased insulin resistance compared with obese mice placed in ambient air. This finding suggests a possible inflammatory role for $\mathrm{PM}_{2.5}$, which may increase insulin resistance, thus contributing to type 2 diabetes mellitus. Other studies have found that patients with diabetes mellitus are more susceptible to adverse events from pollution, although only a few have linked pollution with diabetes prevalence.

"With diabetes care costing the US over $\$ 100$ billion per year and rising similarly around the world, our findings point in a new direction that is worthy of study and of the attention of public health officials, clinicians and policy makers," notes Pearson.

"Obviously, reduction in exposure is a key public health measure," adds senior investigator John S. Brownstein, an epidemiologist in the Informatics Program at Children's Hospital Boston, and concludes, "the findings highlight the need for further research on the inflammatory pathway and the consideration of therapeutic approaches to reduce pollution-induced inflammation."

Linda Koch

Original article Pearson, J. F. et al. Association between fine particulate matter and diabetes prevalence in the United States. Diabetes Care doi:10.2337/dc10-0698 